\title{
Analgosedation: The use of Fentanyl Compared to Hydromorphone
}

\author{
Hahnl Choi ${ }^{1}$, Sara Radparvar ${ }^{1}$, Samuel L. Aitken², Jerry Altshuler ${ }^{3}$ \\ 1 Mount Sinai Hospital, New York, NY, USA \\ 2 University of Texas, MD Anderson Cancer Center, Houston, TX, USA \\ 3 Hackensack Meridian Health JFK Medical Center, Edison, NJ, USA
}

\begin{abstract}
Background: The 2018 Society of Critical Care Medicine guidelines on the "Prevention and Management of Pain, Agitation/Sedation, Delirium, Immobility, and Sleep Disruption in Adult Patients in the ICU" advocate for protocolbased analgosedation practices. There are limited data available to guide which analgesic to use. This study compares outcomes in patients who received continuous infusions of fentanyl or hydromorphone as sedative agents in the intensive care setting. Methods: This retrospective cohort study evaluated patients admitted into the medical intensive care unit, the surgical intensive care unit, and the cardiac intensive care unit from April 1, 2017, to August 1,2018 , who were placed on continuous analgesics. Patients were divided according to receipt of fentanyl or hydromorphone as a continuous infusion as a sedative agent. The primary endpoints were ICU length of stay and time on mechanical ventilation. Results: A total of 177 patients were included in the study; 103 received fentanyl as a continuous infusion, and 74 received hydromorphone as a continuous infusion. Baseline characteristics were similar between groups. Patients in the hydromorphone group had deeper sedation targets. Median ICU length of stay was eight days in the fentanyl group compared to seven days in the hydromorphone group $(p=0.11)$ and median time on mechanical ventilation was 146.47 hours in the fentanyl group and 122.33 hours in the hydromorphone group $(p=0.31)$. There were no statistically significant differences in the primary endpoints of ICU length of stay and time on mechanical ventilation between fentanyl and hydromorphone for analgosedation purposes. Conclusion: No statistically significant differences were found in the primary endpoints studied. Patients in the hydromorphone group required more tracheostomies, restraints, and were more likely to have a higher proportion of Critical Care Pain Observation Tool (CPOT) scores $>2$.
\end{abstract}

Keywords: analgosedation, sedation, analgesic, pain, agitation

Received: 5 July 2020 / Accepted: 6 July 2021

\section{INTRODUCTION}

Analgosedation, also known as analgesia-first sedation or analgesia-based sedation, describes the practice of targeting pain and discomfort in an intensive care unit (ICU) before utilising a sedative agent. Before adopting a protocolised analgosedation practice, many ICU patients were over sedated and undertreated for their pain. Critically ill patients commonly experience pain at rest and with routine intensive care procedures, such as arterial catheter placement, repositioning, tracheal suctioning, chest tube removal, wound drain removal, and turning, with pain being reported in up to $77 \%$ of ICU patients [1-3]. The practice of analgosedation manages pain while providing light sedation. The 2018 Society of Critical Care Medicine guidelines on the
"Prevention and Management of Pain, Agitation/Sedation, Delirium, Immobility, and Sleep Disruption in Adult Patients in the ICU" advocate for protocol-based analgosedation due to favourable outcomes in reducing sedative requirements, duration of mechanical ventilation, ICU length of stay, and pain intensity. However, specific recommendations on the optimal opioid to use are lacking [2].

Previous studies comparing remifentanil to other analgesics observed positive outcomes with remifentanil due to its rapid onset and offset, allowing for easy titration. Remifentanil based therapy was more effective in providing optimal analgosedation than morphine and allowed for more rapid emergence from sedation, thus allowing faster extubation $[4,5]$. However, the adoption 
of remifentanil as a primary analgesic agent has been limited due to the high cost [6]. Currently, fentanyl is the most commonly used agent for analgosedation in the United States.

There are no studies explicitly comparing fentanyl and alternative agents such as hydromorphone for use in this setting. However, critical pharmacokinetic differences, including the onset of action, volume of distribution and half-life, differ significantly between the two agents.

The purpose of this study was to compare the use of hydromorphone to fentanyl for analgosedation.

The null hypothesis is that there is no difference between fentanyl and hydromorphone concerning the length of stay in the intensive care unit or the time on mechanical ventilation.

\section{METHODS}

This was a retrospective single-centre cohort study evaluating patient-data from The Mount Sinai Hospital in New York, USA for fentanyl (Akorn, Lake Forest, IL, USA) intravenously as a continuous infusion titrated to CPOT between April 1, 2017, and December 31, 2018, and hydromorphone (Purdue Pharma, Stamford, CT, USA) intravenously as a continuous infusion titrated to CPOT between January 1 2018, and August 1, 2018.

Data were collected through electronic health record chart review. In addition, a report of orders for continuous hydromorphone or continuous fentanyl drips was run for the study period, and patients were manually screened for inclusion.

This study was approved by the Institutional Review Board (STUDY-20-01361).

Inclusion criteria: Patients were included if they were:

- Eighteen years or older, and received fentanyl or hydromorphone as a continuous infusion during their ICU stay.

- In the medical, surgical, or cardiac intensive care unit and were mechanically ventilated for more than 24 hours.

Exclusion Criteria: Patients were excluded if they:

- had active seizures

- died within 48 hours of admission

- were chronically ventilated from a long-term care facility with a tracheostomy
- received intravenous push doses of analgesics exclusively

- did not require mechanical ventilation.

Data were collected through a retrospective chart review of electronic medical records. In addition, all Richmond Agitation Sedation Scale (RASS) scores, Critical Care Pain Observation Tool (CPOT) scores, and Confusion Assessment Method (CAM) scores in the intensive care unit and background sedative use and doses were collected for the entirety of fentanyl or hydromorphone infusion time.

The data were presented as two groups:

- Group 1, Fentanyl

- Group 2, Hydromorphone

Background sedatives used in conjunction with fentanyl and hydromorphone were recorded, giving six subgroups. The addition of background sedatives was based on individual Richmond Agitation-Sedation Scale (RASS) and Critical Care Pain Observation Tool (CPOT) scores.

During the study period, the institution changed from using the sedation-agitation scale (SAS) to Richmond Agitation Sedation Scale to assess the level of sedation in patients. Sedation-agitation scale scores were converted to equivalent Richmond Agitation-Sedation Scale scores based on prior literature comparing intrascore translatability and were independently appraised by two clinical pharmacists [7].

The Richmond Agitation-Sedation Scale, Sedationagitation Scale, and Critical Care Pain Observation Tool scores are assessed hourly and with each dose titration during the study. In addition, confusion Assessment Method scores in the ICU were assessed at each change of shift. If patients could reliably communicate their pain, numerical rating scales are used instead of Critical Care Pain Observation Tool scores.

Intubation time was based on the data in the respiratory therapist notes.

Extubation was also collected via respiratory therapist notes or recorded as the patient's time of death, whichever came first.

ICU length of stay was calculated as the time of transfer of care to transfer to the floor or time of death.

The primary endpoints assessed were ICU length of stay and the time on mechanical ventilation. If patients died during their ICU stay, they were assigned 30 days or the total duration time in the ICU before death, whichever was longer. 
Patients were listed as being on mechanical ventilation for thirty days if death occurred during ICU stay or if the patient received a tracheostomy.

Secondary endpoints included:

- the percentage of time within RASS goal

- median RASS

- percentage of time that the patient was Confusion Assessment Method in the CU positive during their stay, percentage of time CPOT $>2$

- need for tracheostomy

- constipation defined as no bowel movement for $>72$ hours

- self-extubation

- the need for restraints.

\section{Statistical Analyses}

The primary outcomes were assessed using a two-sample Wilcoxon rank-sum (Mann-Whitney) test.

Baseline characteristics and secondary endpoints were evaluated using Fisher's exact test for categorical variables and Wilcoxon rank-sum test for continuous variables.

The significance level was set at $\alpha=0.05$

All analyses were performed using Stata v14.1 (StataCorp LP, College Station, Texas).

\section{RESULTS}

From April 1, 2017, to August 1, 2018, 444 patients were screened for inclusion or exclusion, resulting in 177 patients being included in the final analysis.

Seventy-four patients were allocated to the continuous hydromorphone infusion group, and 103 patients were allocated to the continuous fentanyl infusion group.

Forty-eight patients (10.8\%) died within 48 hours of admission to the ICU and were excluded from the study analysis.

Recorded baseline characteristics and demographics are listed in Table 1.

Of the total cohort, $61 \%$ were male.

There were no significant differences in baseline characteristics except for coronary artery disease, which was more common among patients in the fentanyl group. Additionally, more patients in the hydromorphone group had deeper sedation goals and were paralysed during their ICU admission.
Paralytic drugs were used in $10.68 \%$ of patients in the fentanyl group compared to $25.68 \%$ of patients in the hydromorphone group. $(\mathrm{p}=0.014)$

There were no significant differences in APACHE II scores between groups at baseline.

The diagnosis on admission for both groups mainly consisted of sepsis, respiratory, or gastrointestinal causes.

In the fentanyl group, $82.52 \%$ had Child-Pugh Class B or C liver dysfunction compared to $89.19 \%$ in the hydromorphone group.

More patients in the fentanyl group were on concomitant dexmedetomidine compared to the hydromorphone group, $41.75 \%$ and $24.32 \%$, respectively. Concomitant midazolam and propofol use were similar between groups.

Background sedative doses did not significantly differ between groups (Table 2).

For the primary endpoints, the median ICU length of stay was eight (IQR 4 - 15) days in the fentanyl group compared to seven (IQR 5 - 11) days in the hydromorphone group $(\mathrm{p}=0.11)$ and median time on mechanical ventilation was 146.47 (IQR 64.55 - 279.69) hours in the fentanyl group and 122.33 (IQR 70.27 - 204.98) hours in the hydromorphone group $(\mathrm{p}=0.31)$. In addition, a median of 1.23 patients in the hydromorphone group had a Critical Care Pain Observation Tool score greater than 2 compared to 0 in the fentanyl group $(\mathrm{p}<0.001)$ (Table 3).

There were no significant differences in the time that a patient was Confusion Assessment Method -ICU positive, the time within sedation goal, or self-extubation (Table 4).

In the hydromorphone group, $44.59 \%$ of patients required restraints compared to $27.18 \%$ in the fentanyl group ( $\mathrm{p}=0.02)$.

In the hydromorphone group, $20.27 \%$ underwent tracheostomy compared to $7.77 \%$ in the fentanyl group $(\mathrm{p}=0.02)$.

Therefore, the study data upheld the null hypothesis.

\section{- DisCUSSION}

In this single-centre, retrospective study, it was concluded that there are no advantages in using fentanyl over hydromorphone for analgosedation in reducing the duration of mechanical ventilation or ICU length 
Table 1. Baseline Characteristics and Demographics

\begin{tabular}{|c|c|c|c|}
\hline & Fentanyl $(n=103)$ & Hydromorphone $(n=74)$ & p value \\
\hline Age (years)a & $63(53-70)$ & $61.5(50-71)$ & 0.54 \\
\hline APACHE $\|^{a}$ & $23(17-29)$ & $25(19-29)$ & 0.36 \\
\hline \multicolumn{4}{|l|}{ Location $^{b}$} \\
\hline $\mathrm{MICU}$ & $52(50.49)$ & 31 (41.89) & \multirow{3}{*}{0.47} \\
\hline SICU & $39(37.86)$ & $35(47.30)$ & \\
\hline $\mathrm{CICU}$ & $12(11.65)$ & $8(10.81)$ & \\
\hline \multicolumn{4}{|l|}{ Admission Diagnosisb } \\
\hline Sepsis & $24(23.3)$ & $13(17.57)$ & \multirow{7}{*}{0.20} \\
\hline Respiratory & $21(20.39)$ & $23(31.08)$ & \\
\hline Gl & $35(33.98)$ & $23(31.08)$ & \\
\hline Metabolic & $1(0.97)$ & $1(1.35)$ & \\
\hline Hematologic & $4(3.88)$ & $0(0.00)$ & \\
\hline Cardiovascular & $15(14.56)$ & $8(10.81)$ & \\
\hline Other & $3(2.91)$ & $6(8.11)$ & \\
\hline$\%$ Maleb $^{b}$ & $67(65.05)$ & $40(54.05)$ & 0.16 \\
\hline Weight (kg)a & $78.20(62.10-105)$ & $70.55(60.40-85)$ & 0.17 \\
\hline \multicolumn{4}{|l|}{ Race } \\
\hline White & $42(40.78)$ & $24(32.43)$ & \multirow{5}{*}{0.12} \\
\hline Black & $28(27.18)$ & $13(17.57)$ & \\
\hline Hispanic & $23(22.33)$ & $21(28.38)$ & \\
\hline Asian & $6(5.83)$ & $9(12.16)$ & \\
\hline Other/ Unknown & $4(3.88)$ & $7(9.46)$ & \\
\hline \multicolumn{4}{|l|}{ Comorbidities } \\
\hline Pulmonary conditions & $32(31.07)$ & $22(29.73)$ & 0.87 \\
\hline Hypertension & $57(55.34)$ & $42(56.76)$ & 0.88 \\
\hline Diabetes & $41(39.81)$ & $32(43.24)$ & 0.76 \\
\hline Coronary artery disease & $51(49.51)$ & $25(33.78)$ & $0.046^{*}$ \\
\hline Chronic Opioid Use & $40(38.83)$ & $38(51.35)$ & 0.13 \\
\hline \multicolumn{4}{|l|}{ Child Pughb } \\
\hline Class A & 18 (17.48) & $8(10.81)$ & 0.28 \\
\hline Class B or C & $85(82.52)$ & 66 (89.19) & \\
\hline Alcohol Withdrawal & $7(6.8)$ & $1(1.35)$ & 0.87 \\
\hline \multicolumn{4}{|l|}{ RASS Goals } \\
\hline 0 to -2 & 97 (96.04) & 60 (82.19) & \multirow{4}{*}{$0.01^{*}$} \\
\hline-2 to -3 & $1(0.99)$ & $5(6.85)$ & \\
\hline-3 to -4 & $2(1.98)$ & $3(4.11)$ & \\
\hline-4 to -5 & $1(0.99)$ & $5(6.85)$ & \\
\hline Paralysis & $11(10.68)$ & $19(25.68)$ & $0.014^{*}$ \\
\hline
\end{tabular}

Abbreviations: APACHE, Acute Physiology and Chronic Health Evaluation; CICU, cardiac intensive care unit; Gl, gastrointestinal; MICU, medical intensive care unit; RASS, Richmond Agitation Sedation Scale; SICU, surgical intensive care unit; median (interquartile range). bN (\%). * Indicates significant difference.

Table 2. Background Sedative Use and Doses

\begin{tabular}{lccc} 
& Fentanyl $(\mathbf{n}=\mathbf{1 0 3})$ & Hydromorphone $(\mathbf{n}=\mathbf{7 4})$ & p value \\
Midazolam & $47(45.63)$ & $30(40.54)$ & 0.54 \\
Propofolb $^{b}$ & $53(51.46)$ & $42(56.76)$ & 0.54 \\
Dexmedetomidine & $43(41.75)$ & $18(24.32)$ & $0.02^{*}$ \\
Propofol (mg/kg/day) ${ }^{a}$ & $16.17(5.035-33.415)$ & $13.52(7.205-27.08)$ & 0.67 \\
Midazolam (mg/day) & $33.06(16.3375-44.6275)$ & $22.76(13.375-52.755)$ & 0.43 \\
Dexmedetomidine (mcg/kg/day) & $7.24(2.51-11.42)$ & $5.10(1.79-8.97)$ & 0.37 \\
\hline
\end{tabular}


Table 3. Primary Outcomes

\begin{tabular}{lccc} 
& Fentanyl $(\mathbf{n}=\mathbf{1 0 3})$ & Hydromorphone $(\mathbf{n}=\mathbf{7 4})$ & $\mathbf{p}$ value \\
ICU Length of Stay (days) & & $7(5-11)$ & 0.11 \\
Time on Mechanical Ventilation (hours) $^{\mathrm{a}}$ & $146.47(64.55-279.69)$ & $122.33(70.27-204.98)$ & 0.31 \\
\hline
\end{tabular}

Abbreviations: ICU, intensive care unit; a Median (interquartile range).

Table 4. Secondary Outcomes

\begin{tabular}{|c|c|c|c|}
\hline & Fentanyl $(n=76)$ & Hydromorphone $(n=47)$ & p value \\
\hline CPOT $>2,(\% \text { of time })^{a}$ & $0(0-0)$ & $1.23(0-5.56)$ & $<0.001^{*}$ \\
\hline CAM-ICU Positive, (\% of time)a & $0(0-7.14)$ & $0(0-19.64)$ & 0.82 \\
\hline Time Within Sedation Goal, (\% of time)a & $63.16(29.27-87.37)$ & 79.69 (35.38- 88.42) & 0.43 \\
\hline Self-Extubation ${ }^{b}$ & $6(5.83)$ & $9(12.16)$ & 0.17 \\
\hline Need for Restraintsb & $28(27.18)$ & $33(44.59)$ & $0.02 *$ \\
\hline Tracheostomyb & $8(7.77)$ & $15(20.27)$ & $0.02 *$ \\
\hline Constipation ${ }^{b}$ & $40(38.83)$ & $38(51.35)$ & 0.13 \\
\hline
\end{tabular}

Abbreviations: CPOT, critical care pain observation tool; CAM-ICU, confusion assessment method for the intensive care unit; >, greater than; \%, percentage; median (interquartile range). bN (\%). * Indicates significant difference

of stay. During the study period, as a result of a fentanyl drug shortage, the hospital changed its primary sedative from fentanyl to hydromorphone in intensive care units.

These data are not consistent with prior studies, which suggested a benefit in opioid analgesics with a shorter half-life concerning the duration of mechanical ventilation $[4,5]$.

It remains unclear why patients receiving fentanyl as a continuous infusion had a numerically longer mechanical ventilation duration than hydromorphone in our study. Of note, patients receiving IV pushes of hydromorphone exclusively were not included for analysis. The longer duration of action provided by hydromorphone may allow patients to maintain hydromorphone boluses for analgosedation. Because of this, patients in the fentanyl arm may have included more opioid-naive patients or required lower analgesic doses. Therefore, opioid-naive patients who could be maintained on hydromorphone boluses arm may have been excluded for analysis. Numerically ICU length of stay and time on mechanical ventilation was longer in the fentanyl group compared to the hydromorphone group. However, this was not statistically significant. For secondary endpoints evaluated, the hydromorphone cohort had a significantly higher median of patients with a percentage of time that CPOT was greater than 2, need for tracheostomy, and need for restraints. Notably, more patients in the hydromorphone group required paralysis and more profound sedation; analyses to adjust for these differences were not performed. A change in ICU practice due to a hospital-wide initiative to improve timely tracheostomy rates may have driven the higher incidence of tracheostomy in the hydromorphone group compared to the fentanyl group.

The practice of analgosedation, in which an analgesic is used as the primary agent for pain control and sedation, offers reduced pain intensity, ICU length of stay, duration of mechanical ventilation, and sedative requirements [2]. Data are available demonstrating favourable effects of remifentanil over morphine owed to remifentanil's faster onset and offset, but remifentanil may not be available at many institutions due to its high cost [4-6]. Small studies have also compared remifentanil to fentanyl in paediatric and neonatal mechanically ventilated patients with mixed results. [8,9] However, these data may not be generalisable to our standard adult ICU population. Furthermore, a study was performed to evaluate other opioids for analgosedation due to a remifentanil shortage in which remifentanil was found to be favourable with regard to faster time to extubation. However, this study evaluated multiple different opioids with differing pharmacokinetics, leading to ambiguity about which specific opioid agent could have led to these result differences [10]. Currently, there is a paucity of literature directly comparing fentanyl to hydromorphone for analgosedation in adult ICU patients. Fentanyl offers a faster onset and offset compared to hydromorphone [11-13]. Additionally, the faster onset of fentanyl is owed to its greater degree of lipophilicity compared to hydromorphone allowing for more rapid distribution and ability to cross the blood-brain barrier. While this may offer benefits in terms of titratability, it may lead to variability in the medication half-life due to the potential for accumulation into the adipose and other lipid-rich tissue. This may lead to a more clinically significant change in 
medication half-life in those who have been on a fentanyl infusion for a more extended period [14]. In this present study, we did not evaluate any body mass index (BMI) differences between groups. It remains uncertain if this factor may have led to any differences in our outcomes. Additionally, at baseline, most of our study population had Child-Pugh class B or C liver dysfunction. While all opioids are metabolised hepatically, fentanyl and hydromorphone undergo different pathways. Unlike fentanyl, hydromorphone undergoes phase 2 glucuronidation. While this would generally imply that hydromorphone would less likely be affected by hepatic dysfunction, available literature seems to imply that severe hepatic impairment may increase systemic concentrations of hydromorphone to a greater extent than fentanyl. Although the two groups did not significantly differ in terms of liver dysfunction at baseline, this may have played a role in the clearance of these opioid analgesics and affected hydromorphone clearance to a greater extent than fentanyl [15-17].

The limitations of this study include its retrospective design limiting the available information for the patients collected. Some data collected, including RASS, CPOT, and CAM-ICU, were determined by nurses allowing for interpatient variability in the documentation of these data collection points. Additionally, a switch from SAS to RASS during this study in which a conversion from SAS to RASS was performed for this study and overlap between these sedation scales could not be thoroughly accounted for. Therefore, any nursing challenges with adjusting to this conversion could not be accounted for. Additionally, we did not evaluate the effects of adjunct enteral opioids. However, this is not standard practice at our institution and should not significantly affect results.

Furthermore, the decision to use either opioid boluses or continuous infusion was attending specific and not standardised across different ICUs. It remains unclear if opioid requirements differed between groups as analgesic doses were not collected. Because these infusions are titrated to effect using CPOT or RASS, differences in dose requirements are less likely to impact this study's results significantly. Finally, this study included CICU, MICU, and SICU patients who may introduce variability in pain source and analgesic requirements. However, given that the patients were evenly distributed between the CICU, MICU, and SICU in both groups, outcomes evaluated should not differ between fentanyl and hydromorphone. In addition, in this institution, the CICU does not include cardiothoracic surgery. Therefore, fentanyl or hydromorphone drips were utilised as analgosedation, likely in the setting of critical illness-related pain rather than post-cardiac surgery-related pain. Furthermore, preliminary data suggest a similar incidence of pain in MICU and SICU populations, and any evaluation of specific ICU populations in this current study would be unlikely to affect results significantly [17]. More extensive, more robust studies are needed to conclude if there is an advantage to one of these agents over the other.

\section{CONCLUSION}

This observational study found no statistically significant differences between fentanyl and hydromorphone concerning the primary endpoints of ICU length of stay and time on mechanical ventilation. Numerically, hydromorphone was associated with approximately 22 hours less time on mechanical ventilation; however, this was not significant. This study did find a significant difference in the need for tracheostomy, restraints, and percentage of time that CPOT was greater than 2, favouring the fentanyl group, which may have been an incidental finding or due to a change in practice within our ICUs. It remains unclear if hydromorphone offers benefit as an agent for analgosedation over fentanyl in ICU length of stay and time on mechanical ventilation. However, given the comparable findings between hydromorphone and fentanyl in this study, one can conclude that either agent may be used for analgosedation without significant clinical outcomes differences. Therefore, it may be reasonable to choose an analgesic agent based on availability in the setting of drug shortages and cost. This is the first study to evaluate the clinical implications of changing primary analgesic agents in the ICU in the setting of a drug shortage. Due to the 2019 SARS-CoV2 pandemic's impact on critically ill patient volumes and supply chain interruptions, many institutions have needed to change their primary analgesic agents. Based on the findings of this study, practitioners may feel more confident in making the transition from one opioid to another for analgosedation based on drug availability. Larger and well-controlled studies are needed to confirm any significant differences between the two agents. 


\section{CONFLICT OF INTERESTS}

The author(s) declared no potential conflicts of interest concerning the research, authorship, and publication of this article.

\section{FUNDING}

The author(s) received no financial support for the research, authorship, and publication of this article.

\section{REFERENCES}

1. Devabhakthuni S, Armahizer M, Data J, et al. Analgosedation: A paradigm shift in intensive care unit sedation practice. Ann Pharmacother. 2012;46(4):530-40.

2. Devlin JW, Skrobik Y, Gélinas C, et al. Clinical practice guidelines for the prevention and management of pain, agitation/ sedation, delirium, immobility, and sleep disruption in adult patients in the ICU. Crit Care Med. 2018;46:e825-73.

3. Faust AC, Rajan P, Sheperd LA, et al. Impact of an AnalgesiaBased Sedation Protocol on Mechanically Ventilated Patients in a Medical Intensive Care Unit. Anesth Analg. 2016;123(4):9039.

4. Dahab AA, Grabner T, Rehak PH, et al. Remifentanil versus morphine analgesia and sedation for mechanically ventilated critically ill patients: a randomised double-blind study. Anesthesiology. 2004;101:640-646.

5. Breen D, Karabinis A, Malbrain M, et al. Decreased duration of mechanical ventilation when comparing analgesia-based sedation using remifentanil with standard hypnotic-based sedation for up to 10 days in intensive care unit patients: a randomised trial. Crit Care. 2005;9:R200-R210.

6. Wolters Kluwer Clinical Drug Information, Inc. (Lexi-Drugs). Wolters Kluwer Clinical Drug Information, Inc.; February 20, 2020.
7. Khan BA, Guzman O, Campbell NL, et al. Comparison and Agreement Between the Richmond Agitation-Sedation Scale and the Riker Sedation-Agitation Scale in Evaluating Patients' Eligibility for Delirium Assessment in the ICU. Chest. 2012;142(1):48-54.

8. Akinci SB, Kanbak M, Guler A, et al. remifentanil versus fentanyl for short-term analgesia-based sedation in mechanically ventilated postoperative children. Paediatr Anaesth. 2005;15(10):870-8.

9. Welding L, Oberthuer A, Junghaenel S, et al. Remifentanil/ midazolam versus fentanyl/midazolam for analgesia and sedation of mechanically ventilated neonates and young infants: a randomised controlled trial. Intensive Care Med. 2012;38(6):1017-24.

10. Klaus, DA, de Bettignies, AM, Seemann, $R$, et al. impact of a remifentanil supply shortage on mechanical ventilation in a tertiary care hospital: a retrospective comparison. Crit Care. 2018;26;22(1):267

11. Purdue Pharma. (2008). Hydromorphone [package insert]. Stamford, CT.

12. Akom, Inc. (2012). Fentanyl [package insert]. Lake Forest, IL.

13. Peng PW, Sandler AN. A Review of the Use of Fentanyl Analgesia in the Management of Acute Pain in Adults. Anesthesiology. 1999 Feb;90(2):576-99.

14. Smith HS. Opioid Metabolism. Mayo Clin Proc. 2009; 84(7): 613-624.

15. Bosilkovska $\mathbf{M}$, Walder $B$, Besson $M$, et al. Analgesics in patients with hepatic impairment: pharmacology and clinical implications. Drugs. 2012;72(12):1645-69.

16. Soleimanpour H, Safari S, Shahsavari Nia K, et al. Opioid Drugs in Patients With Liver Disease: A Systematic Review. Hepat Mon. 2016;16(4): e32636.

17. Chances G, Sebbane M, Barbotte E, et al. A prospective study of pain at rest: incidence and characteristics of an unrecognised symptom in surgical and trauma versus medical intensive care unit patients. Anesthesiology. 2007 Nov;107(5):858-60. 